\title{
Application of Improved BP Neural Network Algorithm in Hydrocarbon Identification of Salinity Mud Invasion Reservoirs
}

\author{
Lixiang Feng ${ }^{1}$, Wang Dan ${ }^{2}$, Rongchao Cheng ${ }^{3}$ \\ ${ }^{1}$ China University of Geosciences, Beijing, 100083, China \\ ${ }^{2}$ Research Institute of Petroleum Exploration and Development, Beijing, 100083, China \\ ${ }^{3}$ CNPC Drilling Research Institute, Beijing, 102206, China
}

Keywords: BP Neural Network, Wire Log, Gas and Geochemical Log, Hydrocarbon Identification

\begin{abstract}
Saline solution mud has been extensively used in many oil and gas fields. Its salinity property leads to the difficulty in identifying hydrocarbon using conventional wire logging information due to the lower reservoir resistivity resulted from saltwater invasion. Gas logging and geochemical logging directly reflect reservoir characteristics and thus can be much less affected by mud invasion. With the aid of FORWARD platform, the wire logging is therefore combined with gas logging and geochemical logging to identify hydrocarbon zones by means of BP neural network. To address the problems of lower convergence speed and easy occurrence of local minimum for BP neural network, a momentum term is added in the BP neural network algorithm, and the prediction accuracy of the method has been significantly improved. The method was successfully used in 48 layers of 5 wells in target oilfield. Testing results show that the coincidence rate of hydrocarbon interpretation is up to $93.7 \%$, which proves the feasibility and efficiency of the method, and shows its application prospect in analogous reservoirs.
\end{abstract}

\section{Introduction}

BP (Back-Propagation) neural network became the most widely used neural network model since it was firstly proposed [1][2]. The generally accepted problem of BP neural network is the slow convergence and easy falling into the local minima problem [3]. In this paper, the method of adding a momentum term was used to accelerate the convergence speed and thus improve the prediction accuracy, which can also indirectly avoid falling into local minimum problems.

Most wells of target oilfield use saline mud in drilling, which causes difficulties to identify hydrocarbon using conventional wire logging information affected by Salinity mud invasion [4]. Gas logging and geochemical logging is a direct response to oil and gas, and is contaminated lightly [5]. Therefore, the wire logging, gas logging and geochemical logging data were combined based on the FORWARD platform, using BP neural network to identify hydrocarbon zones [6][7].

In the study oilfield, the BP network structure was built by 10 input neurons, 2 hidden layers, and 5 output neurons. Preferably oil and gas sensitive parameters and representative samples were used to repeat training the neural network, which achieve better identification results for hydrocarbon zones.

\section{Theory of BP Neural Network}

Error back propagation neural network consists of an input layer, hidden layer and output layer. Each layer consists of a plurality of neurons in turn. The neurons are not connected in the same layer, but be connected between each two adjacent layers.

\section{BP Neuron Model}

Neurons are the basic arithmetic unit of neural network, and BP neural network-based algorithm is neuron mode. Neurons are generally nonlinear element with multiple inputs and one output, which usually consists of an internal feedback $(S)$, thresholds $(\theta)$, and characteristic function of neuronal activity. The mathematical expression is: 


$$
\begin{aligned}
& \mathrm{X}=\sum \omega_{\mathrm{i}} x_{i}+s \\
& \mathrm{X}^{\prime}=F\left(X_{i}\right)
\end{aligned}
$$

Where, is the input value; $\omega_{\mathrm{i}}$ is the weights; $\mathrm{X}$ is the total value of input neurons; $\mathrm{X}$ ' is the output value; $F$ is the characteristic function of neuronal activity.

The different functions have different gain characteristics, and here we use S-type nonlinear function after comparison:

$$
F(X)=\frac{1}{1+e^{-(X+\theta)}}
$$

\section{BP Neural Network Model}

A typical form of neural networks is a connection model, which is connected by an independent neurons, the network is divided into several layers. The input layer neurons and output layer neurons are independent without connections, the output of $j$ neuron in the hidden $k$-layer is:

$$
y_{i}^{(K)}=f\left(\sum_{i=1}^{N_{k-1}} W_{i j}^{(k-1)} y_{i}^{(k-1)}-\theta_{j}^{(k)}\right)
$$

Where, $y_{i}^{(K)}\left(j=1,2, \cdots, N_{k} ; k=1,2, \cdots, M\right)$ is the output of $j$ neuron; $W_{i j}^{(k-1)}$ is the connection weights; $\theta_{j}^{(k)}$ is the threshold; $f$ is the transfer function of neurons; $N_{k}$ is the number of $k$-layer neurons; $M$ is the total number of layers.

\section{The Learning Algorithm of BP Neural Network}

The BP Network learning process of three layer structure can be described as follows:

Step1: (Forward propagation to calculate the neuron outputs) First initialize the weights and thresholds using random number, Then input samples, the top-down computation is according to formula (4) to calculate the output of each neuron from the beginning of the input layer .

Step2: (Back propagation to calculate the errors of output layer neurons) The total square error of output layers is as follows:

$$
E=\frac{1}{2} \sum_{k=1}^{P}\left(t_{k}-o_{k}\right)^{2}
$$

Where, $t_{k}$ is the output of sample and $o_{k}$ is the calculation result of the neuron $k$.

Step3: (Connection weights correction) In order to make the calculation convergent, and to make $\mathrm{E}$ tend to minimum, then amend the connection weights and bias values.

$$
\Delta w_{k j}=-\eta \frac{\partial E}{\partial w_{k j}}
$$

Connection weights correction for the output layer neurons is as follows:

$$
\omega_{k j}(n+1)=\omega_{k j}(n)+\Delta \omega_{k j}(n)=\omega_{k j}(n)+\eta \delta_{k} o_{j}
$$

Connection weights correction for the non output layer neurons is as follows:

$$
\omega^{(k-1)}{ }_{i j}(n+1)=\omega^{(k-1)}{ }_{i j}(n)+\eta \delta^{(k)}{ }_{j} O_{j}^{(k)}
$$

Step4: (Loop learning to finalize the network weights) To calculate the current output error of the learning sample I:

$$
e p_{I}=\sum_{j=1}^{P}\left|t_{j I}-o_{j I}\right|
$$

Then calculate the cumulative error as follows:

$$
\varepsilon=\frac{1}{2} \sum_{I=1}^{N} \sum_{j=1}^{P}\left(t_{j I}-o_{j I}\right)^{2}
$$


If the cumulative error is bigger than the termination error $\left(\varepsilon>\varepsilon_{0}\right)$, then repeat the learning; else stop the learning as the process has converged and the connection weights have been set, which can reflect the relationship between input and output layers in the allowed error range.

\section{Algorithm Improvement}

There are some deficiencies in practical applications of basic BP neural network algorithm [8]. Its widely recognized problem is the slow convergence and easy falling into local minimum. In this paper, a method of adding momentum term is proposed to improve the convergence speed of BP neural network algorithm.

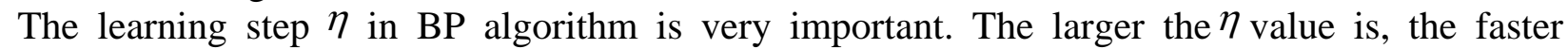
network convergence shows. But oversize $\eta$ can cause oscillation instability, smaller $\eta$ leads to lower network convergence. The best way to resolve this contradiction is to introduce the momentum term as follows:

$$
\Delta \omega_{i j}(n+1)=\alpha \Delta \omega_{i j}(n)+\eta \delta_{j}(n) o_{j}
$$

Where, $\alpha$ is momentum term, usually an integer. The amount of $(n+1)$ correction should be maintained on the correction amount of the $(n)$ correction to some extent, so changes in the law of inertia keep in a way of conservation. Adding momentum in BP algorithm can not only speed up the convergence rate, but also can make learning to avoid falling into local minima.

\section{Application of Improved BP Neural Network Algorithm in Hydrocarbon Identification of Salinity Mud Invasion Reservoirs}

In the research oilfield, Saline solution mud has been extensively used. Its salinity property leads to the difficulty in identifying hydrocarbon using conventional wire logging information due to the lower reservoir resistivity resulted from saltwater invasion. Gas logging and geochemical logging directly reflect reservoir characteristics and thus can be much less affected by mud invasion. With the aid of FORWARD platform, the wire logging is therefore combined with gas logging and geochemical logging to identify hydrocarbon zones by means of BP neural network.

Based on the above-mentioned theory, the improved BP neural network algorithm is applied to the target oilfield, using the wire logging, gas logging and geochemical logging data together to establish a corresponding optimum network structure. The process of building a network structure is divided into three parts as follows, 1) selection of learning parameters and samples and preprocessing, 2) establishment of network structure, 3) back generation forecast of learning samples[9] [10]:

Step1: (Selection and pre-processing of learning parameters and samples) Through the analysis of logging curves, and considering the sensitive degree of oil, gas, and water, after multiple neural network trials, finally three wire logging parameters: RT (resistivity of the formation), $\mathrm{SH}$ (shale content of the formation), DEN(density of the formation) and seven surface logging data C1(C1 component of gas logging), S1(liquid hydrocarbon component of geochemical logging), S2(heavy hydrocarbon component of geochemical logging), QT(total hydrocarbon of gas logging), $\mathrm{Pg}$ (sum of S0, S1, and S2, S0 is the gaseous hydrocarbon component of geochemical logging), C1/QT, S1/Pg were determined, which are sensitive to hydrocarbons[11].

According to the conventional well logging data interpretation results, oil layer, water-oil layer, oily water layer, water layer, dry layer, a total of five neurons are set as the network's output layer. In order to realize the digital processing of neural network, the 5 types of reservoir layer are set code as follows: oily water layer(0.1 $0.1 \quad 0.1 \quad 0.1 \quad 0.9)$, water-oil layer(0.1 $0.1 \quad 0.1 \quad 0.9 \quad 0.1)$, oil

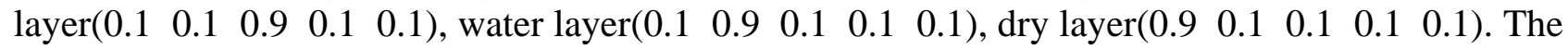
neuron activation function is sigmoid function, and the asymptotic values are 1 and 0 . In order to avoid non convergence of the learning algorithm, the code was set as 0.1 and 0.9 . 


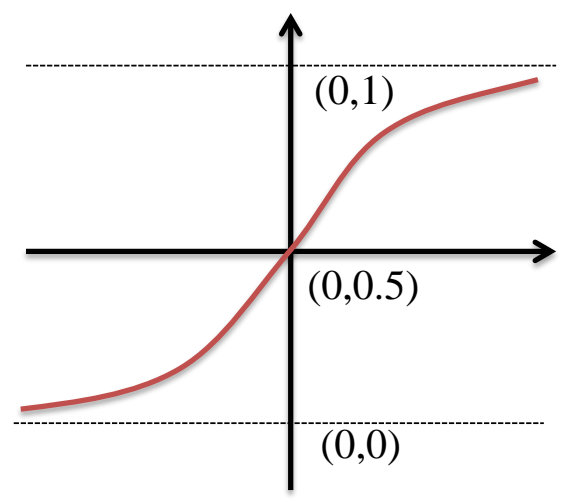

Fig.1: Sigmoid function

Then we choose 32 learning samples corresponding to the previously described five type layers, these 32 samples are from well $* 101$, well $* 102$, well $* 104$, which were confirmed by oil testing data. The numerical value of each input parameter of learning samples have different order of magnitude, so the normalization should be done before calculation, the normalization method used in this paper is as follows:

$$
x=\frac{X_{o}-x_{\min }}{x_{\max }-x_{\min }}
$$

Where, $x_{o}$ is the original value of the input layer, $x$ max is the maximum value, and $x$ min is the minimum value of the input layer.

Step2: (Determining the network structure)Research on the number of hidden layers using 1 or 2 try method, and ultimately determine the number of hidden layers is 2 after samples learning, the numbers of the hidden layer are 5 and 8 . So in the target oilfield, the BP network structure was build by 10 input neurons, 2 hidden layers, 5 output neurons, also the connection weights and thresholds between neurons are saved in order to achieve reservoir prediction.

Step3: (Back generation forecast of learning samples)To test the accuracy and feasibility of the algorithm, using the trained weights to predict the own learning samples, the predicted results are $100 \%$ consistent.

\section{Test Results in Target Oilfield}

Based on FORWARD platform, Well*101, Well*102 were processed and analyzed using improved BP neural network algorithm, figure 2 shows the flow and figure 3 shows the typical well predicted results. A total of 48 layers of 5 wells were processed, 45 layers were confirmed by oil testing data, so the consistence rate is up to $93.7 \%$ [12]. 


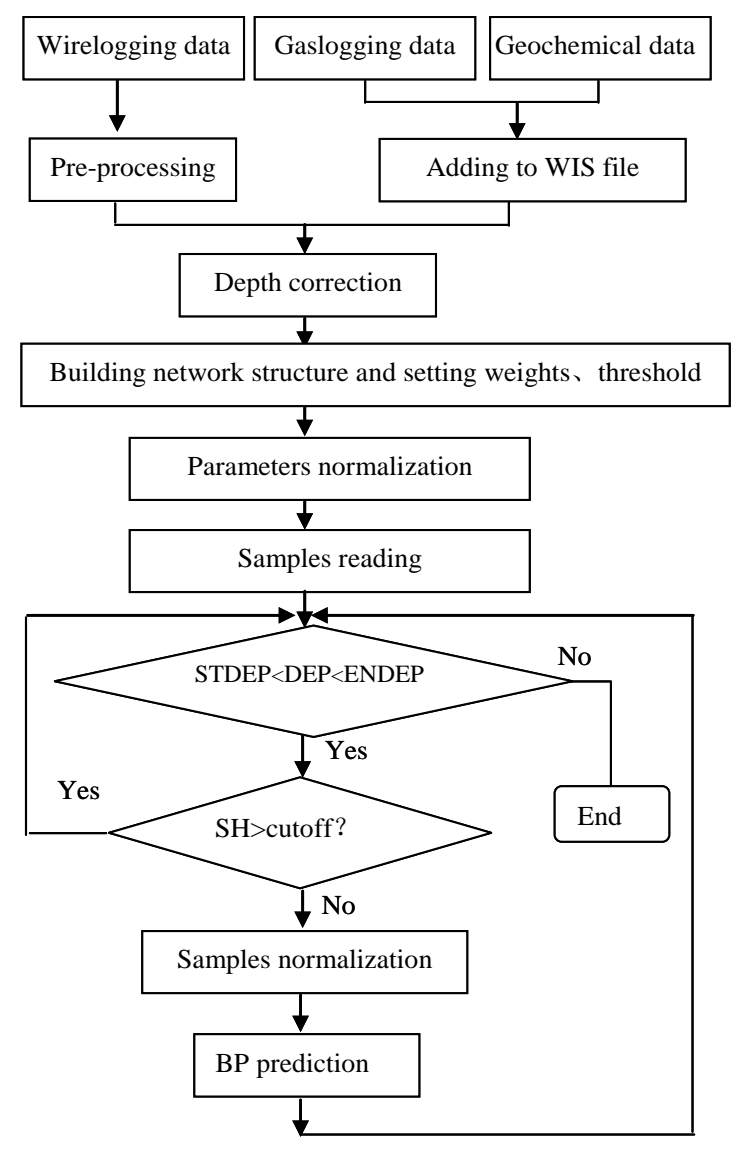

Fig.2: Flow chart of hydrocarbon identification using BP Neural Network Algorithm based on FORWARD platform

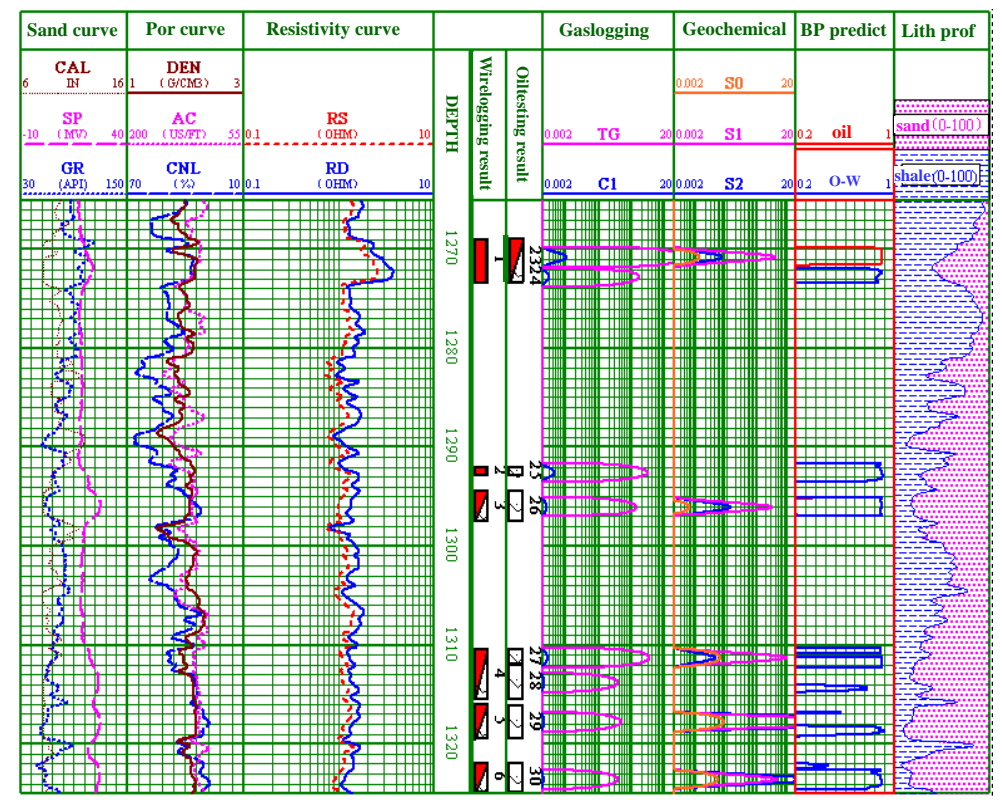

(a) 


\begin{tabular}{|c|c|c|c|c|c|}
\hline $\begin{array}{c}\text { Well } \\
\text { name }\end{array}$ & Depth（m) & $\begin{array}{l}\text { Surfacelogging } \\
\text { result }\end{array}$ & $\begin{array}{l}\text { Wirelogging } \\
\text { result }\end{array}$ & $\begin{array}{l}\text { Testing } \\
\text { result }\end{array}$ & $\begin{array}{c}\text { Prediction } \\
\text { result }\end{array}$ \\
\hline \multirow{8}{*}{ *101 } & $1269.5-1272.0$ & Oil & Oil & \multirow[b]{2}{*}{ Water-oil } & Oil \\
\hline & $1272.0-1273.5$ & Water-oil & Oil & & $\begin{array}{l}\text { Oily } \\
\text { water }\end{array}$ \\
\hline & $1292.0-1293.0$ & 1 & Oil & \multirow{2}{*}{$\begin{array}{l}\text { Oily } \\
\text { water }\end{array}$} & $\begin{array}{l}\text { Oily } \\
\text { water }\end{array}$ \\
\hline & $1294.5-1297.5$ & Oil & Water-oil & & $\begin{array}{l}\text { Oily } \\
\text { water }\end{array}$ \\
\hline & 1310.5-1312.0 & Oil & \multirow{2}{*}{ Water-oil } & \multirow{4}{*}{$\begin{array}{l}\text { Oily } \\
\text { water }\end{array}$} & Oily \\
\hline & $1312.0-1315.5$ & Oil & & & water \\
\hline & 1316.1-1319.5 & Oil & Water-oil & & $\begin{array}{l}\text { Oily } \\
\text { water }\end{array}$ \\
\hline & 1322.0-1325.0 & Oil & Water-oil & & $\begin{array}{l}\text { Oily } \\
\text { water }\end{array}$ \\
\hline
\end{tabular}

(b)

Fig.3: Interpretation results of well*101 (fig a is the result profile of well *101 based on FORWARD platform; fig b is the interpretation results comparison)

\section{Conclusions}

Based on FORWARD platform, improved BP neural network was used to identify hydrocarbon zones with comprehensive information from wire logging, gas logging and geochemical logging. Actual production tests proved that the method has great value in the target oilfield drilled with saline solution mud. Throughout the learning, researchers conclude some issues still need to better addressed, such as how to extract representative parameters and establish accurate samples.

\section{References}

[1] Gao Jun, Artificial neural network theory and simulation examples, Machinery Industry Press, 2003.8.

[2] Jiang Zongli, Introduction to artificial neural networks, Higher Education press, 2001.8.

[3] Pao,Yoh-h, Adaptive pattern recognition and neural networks, Science Press,1992.1.

[4] Ouyang Jian, Quantitative study of dual laterolog response to oil/gas formation invaded by high Salinity Mud Filtrate in Bohai Gulf. WLT, 1998, 22(4): 256-260.

[5] Liang Congjun, Exactly deciding hydrocarbon reservoir through combining well logs with geological log data, Natural Gas Industry, 2004, 23(4): 52-56.

[6] Yang Hui, Application of BP neural net in lithologic identification of tight sandstone gas reservoirs, Petroleum Geophysics, 2013, 11(1): 39-42.

[7] Yang Sitong, Identification of hydrocarbons in low-porosity and low-permeability reservoirs by integration of surface log data with wire log information, Oil\& Gas Geology, 2007, 28(3): 408412.

[8] MATLAB Chinese Forum, 30 case studies of MATLAB neural network, Beijing University of Aeronautics and Astronautics Press, 2010: 1-10.

[9] Zou Wei, Application of BP neural network in tight sandstone reservoirs, Progress in Exploration Geophysics, 2006, 29(6):428-432.

[10] Jia Lingxiao, Application of improved BP neural network to fracture prediction, Journal of Engineering Geophysics, 2012, 9(3): 302-307. 
[11] Yong Shihe, Zhang Chaomo, Logging data processing and comprehensive explanation [M], Dongying: Petroleum University Publishing House, 1996.

[12] Ouyang Jian,Geological analysis and reservoir quantitative evaluation using logging data, Petroleum Industry Press, 1999. 